\title{
NOTES RECUEILLIES AU COURS DE LA CONFÉRENCE DU PROFESSEUR LAGROT*
}

\author{
le $2 \mathrm{I}$ avril $\mathrm{i} 970$
}

\begin{abstract}
“Vanité du mot Expérience. L’expérience n'est pas expérimentale. On ne la provoque pas. On la subit. Plutôt patience qu'expérience. Nous patientons, plutôt, nous subissons. ”)
\end{abstract}

A. Camus.

Les lésions des mains consécutives à un emploi professionnel fautif des rayons $\mathrm{X}$ sont différentes de celles qui peuvent succéder à une application thérapeutique : les premières touchent l'utilisateur, médecin ou ingénieur, les secondes affectent le malade traité; les premières sont causées par l'usage de la radioscopie à petites doses longtemps répétées chez les médecins, et elles sont d'évolution lente, insidieuse et chronique; ou bien elles sont accidentelles chez l'ingénieur par le fait d'une irradiation brutale erronée, et elles sont aiguës comme une brûlure thermique. Les secondes font suite à un traitement radiothérapique (pour cancer, pour verrue, etc.) mal conduit, ou plus rarement à des examens radioscopiques ou radiographiques à doses excessives; en général dans ces cas, une brûlure aiguë succède à une évolution chronique plus ou moins retardée.

Mais ce qui est commun aux deux cas est l'aboutissement à la dégénérescence cancéreuse, en général tardive, et infiniment plus fréquente dans les formes professionnelles chroniques : certaine, peut-on dire, si le temps lui en est laissé.

Mon expérience, en ce qui concerne les radiodermites professionnelles des mains, s'est faite en très grande majorité sur des médecins qui ont travaillé sous écran scopique pendant des années pour réduire des fractures, rechercher des corps étrangers, procéder à des investigations pulmonaires systématiques, à des radiodiagnostics digestifs, vasculaires, etc. Enfin, ma statistique comporte ro4 cas opérés en vingt et un ans (mais j'en ai bien observé le triple) avec 179 mains opérées. ro cas seulement étaient d'étiologie post-thérapeutique, non professionnelle. Dans les 94 cas professionnels, il n'y avait que 4 ingénieurs brûlés par irradiation aiguë, mais 90 médecins (exactement 42 chirurgiens ou orthomédistes, 32 médecins généralistes ou phtisiologues, 14 radiologues, 2 infirmières). L'énorme majorité de chirurgiens et de médecins par rapport aux radiologues s'explique par cette notion que ces derniers sont instruits des dangers des rayons qu'ils manipulent, ayant reçu un enseignement de physique, tandis que les autres médecins manient leur appareil avec une imprudente ignorance,

* Professeur de Clinique Chirurgicale - Chirurgien des Hôpitaux - Membre Associé national de l'Académie de Chirurgie, 84, rue Alfred-Dumeril, 3 I - Toulouse.

RADIOPROTECTION, VOL. $6-\mathrm{N}^{\circ} 3$ 
insconscience ou insouciance; les lésions insidieusement lentes et chroniques, indolores, n'apparaissant qu'à retardement au bout de cinq, dix, vingt ans, leur méfiance n'est point éveillée; persuadés de leur immunité, ils accumulent, à crédit, des doses de rayons dont la sommation est enregistrée par les téguments jusqu’à des totaux invraisemblables : sous le régime habituel de scopie $(80 \mathrm{kV}, 3 \mathrm{~mA}$, filtre $\mathrm{Al} \mathrm{de} 0,5 \mathrm{~mm}$ ), avec une distance foyer-peau de $40 \mathrm{~cm}$, la main qui réduit une fracture reçoit de 6 à 7 röntgens par minute. Ce débit d'exposition est à rapprocher de la valeur maximale admissible donnée par les normes de radioprotection : is röntgens en I $_{3}$ semaines consécutives.

En y réfléchissant, on est frappé des désastres que l'on provoque lorsque, pour mieux voir, on utilise le régime de graphie avec $80 \mathrm{kV}$ et de ro à $\mathrm{I} 2 \mathrm{~mA}$, et lorsque l'ampoule se trouve à quelques centimètres du malade et du chirurgien. Les doses absorbées peuvent donc être énormes lorsqu'un opérateur a pu passer des heures entières à extraire des éclats sous écran, comme cela s'est produit pendant la dernière guerre.

La lésion radiodermique survient-elle immanquablement au-delà des doses dites raisonnables? Pas forcément, en raison de variations individuelles de sensibilité. Ces normes donnent un avertissement de prudence au-delà desquelles un risque est pris, et en deçà desquelles il est conseillé de se tenir. De même, on pourrait penser qu'il y a une relation entre les doses absorbées et l'apparition ou le degré des lésions. Ici encore le déterminisme n’est ni évident, ni constant, quand nous relevons, parmi d'autres, des observations comme celles qui suivent. Certains de nos opérés ont pu faire le compte des doses qu'ils " encaissèrent » :

- un chirurgien belge prend annuellement 600 à I $500 \mathrm{R}$ et totalise $17842 \mathrm{R}$ en 4428 minutes sur vingt-huit ans, avec une moyenne de 729 séances, ce qui représente une exposition annuelle de 48 minutes équivalant à une dose de rayonnement de $595 \mathrm{R}$ : il ne présente cependant qu'une radiodermite relativement bénigne, avec un épithélioma encore intra-épidermique;

- un orthopédiste belge, pendant sept ans et trois mois, s'exposant aux radiations pendant 2862 minutes, reçoit 4000 à $6000 \mathrm{R}$ par an, prenant au total l'invraisemblable quantité de $35775 \mathrm{R}$ : or, il n'a qu'une radiodermite simple avec hyperkératose, sans dégénérescence maligne;

- un chirurgien français totalise $19000 \mathrm{R}$ en cinq ans et il a fonctionné en intensités de graphie, pour mieux y voir, pendant deux ans : il n'a qu'une radiodermite simple hyperkératosique.

De tels exemples, qui ne sont pas exceptionnels, tendraient à laisser croire qu'il n'y a pas, pour de tels cas, de “ justice immanente ». En réalité, pas toujours seulement. Et ils tendraient à laisser croire aussi qu'il n'y a pas forcément de rapport entre les quantités de rayonnement ionisant " encaissées » et l'importance des lésions. Mais il faut retenir ces lignes de Tubiana et alii (1963) : " Les doses relativement élevées, évaluées entre 100 et 10000 rads semblent de $10 \%$ et après des délais de quinze ans ou plus, puisque des cancers sont apparus sur des cicatrices de radiodermites plus de quarante ans après l'irradiation ". Or, dans nos cas chroniques professionnels, il s'agit non plus de radiodermites cicatrisées, périmées, mais en pleine évolution, en état actif permanent et renouvelé d'excitation cellulaire. 
Aux exemples euphorisants évoqués plus haut, il faut opposer d'autres constatations moins rassurantes :

- un médecin, vraiment innocent..., prend son ampoule à la main pour la diriger : un épithélioma se déclare (et pour comble de malchance, il est traité par radiothérapie !)

- plusieurs chirurgiens, déjà atteints de lésions à évolution torpide, s'exposent par fait de guerre à un brusque surcroît de scopies : leurs lésions s'étendent, prolifèrent, se transforment.

Dans de tels cas, il a été évident qu'un supplément important et rapide d'irradiation a été la cause de l'aggravation et de la malignisation de lésions latentes et de la survenue de nouvelles atteintes.

Nous pouvons en conclure qu'un usage abusif continu plus ou moins régulier des rayons de scopie cause des lésions à évolution lente et longtemps bénigne; et qu'un surcroît plus ou moins massif d'exposition, accidentel ou brutal, détermine toujours une aggravation des lésions et, en général, leur rapide malignisation.

Toujours dans la discussion des causes adjuvantes, peut-on croire à des prédispositions personnelles aux radio-lésions? Il me paraît impossible de préciser la réalité d'une disposition à une radiosensibilité souvent évoquée. Il n'y a pas de possible mesure d'une telle sensibilité, sinon par la cuisante et trop tardive expérience clinique. Santagostini évoque " d'extraordinaires variations de réceptivité sans accident d'un sujet à l'autre » et " pense de plus en plus qu'il y a là un élément qui nous échappe ").

Mais il me semble qu'existe une certaine différence d'évolutivité selon la " qualité grossière » des téguments atteints; plus grave, plus ulcérative, plus dégénérative sur les peaux fines; plus lente, plus kératosique sur les peaux épaisses. Parfois, l'évolution parut inversée : d'un tégument normal, le développement radiodermique fit une peau scléro-atrophique ou une peau pachydermique avec, sur chacune son évolutivité propre, habituelle, plus sévère ou plus bénigne.

L'influence du rayonnement secondaire a paru certaine dans quelques cas : lésions au niveau des bagues, rôle du matériel métallique d'ostéosynthèse manipulé, et aussi sans doute majoration des lésions de la peau mince du dos de la phalangette prise en sandwich entre le rayon direct et le rayonnement réfléchi de la phalange osseuse immédiatement sous-jacente.

Le rôle parfois nocif de l'amplificateur de brillance paraît méconnu mais réel, et nous avons vu, de son fait, quatre cas d'aggravation de lésions latentes. Donnant une sécurité illusoire, il autorise des abus : " pour voir mieux, écrit SanTAgostrinI, on augmente les millis, on part de $0, \mathrm{I}$, on arrive à $\mathrm{I} \mathrm{mA}$ et plus $)$.

J'ai aussi tenté, dans ma statistique, de préciser certaines autres conditions étiologiques d'apparition des radiodermites de mes opérés.

Relevant d'abord l'âge du patient lors de la survenue des lésions, on note que celles-ci débutèrent en majorité entre 30 et so ans chez les chirurgiens et un peu plus tard chez les médecins généralistes et les radiologues.

En ce qui concerne l'âge au moment de l'opération, la majorité concernait, dans l'ensemble, des patients âgés de 40 à 60 ans, surtout de 50 à 60 ans, aussi bien chez les médecins généralistes que chez les chirurgiens; les radiologues furent en général opérés plus tôt, dans leur quatrième décennie. Faut-il conclure que les symptômes apparaissent plus précocement chez les chirurgiens, mais que ceux-

vol. $6-\mathrm{N}^{0} 3$ 
ci se font opérer plus tard que les autres, parce que plus accaparés par leur profession, leur service, leur clinique ? Et que les radiologues viendraient à l'opération plus tôt, mieux informés, plus instruits de l'action des rayons, plus soucieux du pronostic de leur lésion?

Plus importante semblerait être a priori, l'analyse des délais d'apparition des premières lésions à partir du début de l'emploi des rayons. On peut l'appeler l'incubation. En fait cette analyse révèle que cette durée d'incubation n'a paru être soumise à aucune prévision possible de latence, et tous les délais d'éclosion ont été observés sur nos 104 opérés, entre I et 37 ans. Il est vraisemblable que ce délai est déterminé par l'intensité, la quantité, la répartition des expositions, et d'autres facteurs locaux; mais un déterminisme précis ne peut être établi, et les pratiques différentes des chirurgiens, des médecins généralistes, des radiologues, des techniciens, n'ont pas paru avoir d'influence sur ces délais d'incubation.

Il serait intéressant aussi, d'autre part, de connaître l'ancienneté des lésions depuis leur début jusqu'à l'opération, de chercher à estimer le degré et la rapidité d'évolution du mal, mais intriqués avec la durée de la tolérance des patients, estimation qui ne peut être que moyenne et relative en raison de l'individualité psychique et sociale très variable d'un patient à un autre. Cependant, les différents groupes (chirurgiens, médecins généralistes, radiologues) ont suivi des évolutions moyennes parallèles sur ce point : la majorité de leurs lésions évoluait depuis une dizaine d'années, mais c'est une donnée moyenne, la courbe qui la représente grimpant de la première à la dixième année et redescendant ensuite jusqu'à la vingt-cinquième année.

Notons aussi que malgré l'abstention à retardement, totale ou partielle de l'emploi des rayons, les lésions installées à un certain stade ont poursuivi leur évolution et ont amené les patients à la décision opératoire. Durant ces années de patience, et souvent de passion, bien des repentirs et des velléités de sagesse émergèrent, avec des abandons plus ou moins prolongés de la pratique des rayons : l'évolution de la maladie n'en fut pas arrêtée pour autant, après parfois une rémission. La marche irréversible de cette forme de "machine infernale» doit engager les radiolésés à ne pas attendre le moment imprévu de son explosion.

Les modalités différentes d'emploi des rayons par les chirurgiens, les généralistes, les phtisiologues, les radiologues interviennent-elles dans les effets des irradiations? Pour répondre à cette question, il importait de rechercher les incidences propres à chacune de ces spécialités. Les chirurgiens contractèrent tous leur mal au cours de scopies répétées pour réduction de fracture et pour extraction de corps étrangers sous écran. Les orthopédistes furent particulièrement touchés, et les enclouages sans découverte du foyer de fracture furent, et demeurent spécialement dangereux par la longueur des manipulations et par l'action du rayonnement secondaire provenant du matériel métallique.

Presque toujours la lésion initiale latente ou minime fut exacerbée par un surcroît accidentel d'irradiation du fait d'un excès de travail sous rayons, en cours de guerre le plus souvent, par afflux de blessés dans une offensive ou un bombardement, par manque de radiologues, ou par obligations de faire des scopies par manque de films.

Il ne fut pas rare qu'un traumatisme aggravât la lésion (i 4 cas) d'une façon aiguë, comme par exemple ce fut le cas d'un chirurgien torturé à coups de règle sur les doigts, par la Gestapo, ou la suite d'un malencontreux ponçage de verrue 
ou d'un arrachement de croûtes, ou d'écorchures au cours de jardinage ou d'entomologie, ou du serrage du fil chirurgical de ligature au cours des opérations, fait souvent cité.

Parmi les médecins, les plus atteints furent les phtisiologues, les médecins du travail, les pédiatres, à cause des quantités importantes de rayons prises au cours des examens systématiques pulmonaires de groupe, des transits digestifs, des angiographies de plus en plus pratiquées, des pyélographies, etc.

Chez les radiologues, s'ajoutent les effets des utilisations thérapeutiques défectueuses des rayons, la röntgen- et la curiethérapie, la contactothérapie : un de nos opérés utilisait son doigt comme appui du lobe de l'oreille ou de la narine à irradier. Sans parler de tel médecin qui utilisait à chaque scopie sa propre main comme test de bonne visibilité, de tels autres, nombreux, qui, en scopie, tenaient à deux mains leur écran portatif; de cinq autres, en Italie, qui furent victimes d'une pratique thérapeutique néfaste, périmée j'espère, qui prétendait soigner les troubles trophiques et les dégénérescences des irradiations par la " plésiothérapie "; c'est-à-dire une supplémentaire contactothérapie; en effet, les ulcérations étaient améliorées pendant quelques semaines, mais une véritable explosion apparut ainsi simultanément peu après ce «traitement $\mathrm{du}$ mal par le mal » (ô combien!). L'enfer du radiolésé est pavé de telles bonnes intentions!

Il faut ici dénoncer les regrettables erreurs de diagnostic qui firent, dans dix de nos cas, baptiser eczémas des radiodermites et les traiter (?) par radiothérapie. Bien pire, fut la décision délibérée de traiter par les rayons encore six radiodermites dûment diagnostiquées. Des épithéliomas s'ensuivirent dans presque tous ces cas.

Le róle du radium dans la genèse des lésions est certain dans 6 de nos observations ( $s$ radiologues, I infirmière) par manipulation imprudente de tubes, de moules, d'aiguilles dans la curiethérapie des cancers du col, des lèvres, de la langue, de la peau.

Mais l'imprudence, l'ignorance, l'insouciance ne sont pas seules en cause : il faut aussi incriminer la source des rayons, l'appareillage, la protection, les doses émises, enquête difficile et dont les résultats varient selon les spécialités des utilisateurs atteints. Certains radiologues, en Italie surtout, avaient des appareils vétustes et devaient, en outre, en raison du psychisme latin très personnel de leur clientèle, opérer toujours par eux-mêmes et " prendre du rayon " à longueur de journée. Parmi les médecins, beaucoup avaient des appareils non protégés ou " préhistoriques ». Les chirurgiens, opérant sous écran, perdaient la notion du temps, rapprochaient l'ampoule pour mieux voir, et l'intensité augmentait en raison inverse du carré de la distance, et bien pire, deux d'entre eux avouèrent ingénûment avoir travaillé, pendant des années en régime de graphie, pour mieux voir. De plus chez les chirurgiens, le tégument est fragilisé par le brossage traumatisant, privé de sa protection sébacée par le savonnage et les gants de caoutchouc y entretiennent une humidité propice au mordançage des rayons. Dans nos observations la pratique radiologique de ces chirurgiens s'était établie entre trois et quarante années, en majorité entre dix et quinze, à un rythme souvent quotidien et pluri-quotidien, parfois deux heures par jour, les circonstances de guerre les y forçant.

vol. $6-\mathrm{N}^{\circ} 3$ 
On a déjà vu que, malgré cela, il n'apparaît pas évident qu'il existe une corrélation précisément déterminée entre l'importance des doses reçues et la gravité des lésions. Il faut cependant retenir le lourd tribut payé par les orthopédistes, puisque leurs lésions furent trouvées dégénérées dans les trois quarts de leurs cas; encore plus par les phtisiologues, dont les radiodermites étaient cancéreuses huit fois sur neuf, tandis que, chez les généralistes, la dégénérescence existait " seulement" (si l'on ose dire ainsi) que dans les deux tiers des cas. On n'en est pas surpris quand on relève dans les observations des phtisiologues des cadences d'examens systématiques pulmonaires de 7000 , is 000 scopies annuelles, 400 par jour bi-hebdomadaires pendant vingt ans, 40000 en trois mois dans un camp de prisonniers, 150000 dépistages en cinq mois, en Allemagne. Cette fréquence de la cancérisation dans des conditions si particulières, si bien précisées, quasi expérimentales, ne pourrait-elle pas apporter aux biologistes des rayons des éléments susceptibles d'éclairer mieux le déterminisme physique de la dégénérescence cancéreuse radiologique?

Le lecteur voudra bien pardonner ce long chapitre des causes et des conditions. C'était là, me semble-t-il, la meilleure façon de montrer les fautes qu'il ne faut pas faire quand on manie les rayons. Tel était mon but principal. Aussi ne m'appesantirai-je pas sur l'évidence des lésions.

Les doigts atteints sont les quatre derniers, jamais le pouce, sur le dos des deux dernières phalanges et l'ongle, le bout de la pulpe. Les premières lésions apparaissent dans des délais variables, entre cinq et dix ans en moyenne, le plus souvent sur le tégument du dos de la troisième phalange, tégument qui s'amincit et se dessèche, et sous l'ongle qui se strie, puis l'atrophie cutanée s'accompagne du développement d'un fin réseau de vaiseaux, téléangiectasies superficielles. A ce premier stade, qui dure un an environ, l'interruption de la pratique des rayons peut amener la régression des lésions. Sinon, celles-ci évolueront inéluctablement vers une aggravation, sous forme d'extension vers le dos de la deuxième phalange, en général par des verrues hératosiques isolées ou en plaques qui vont en s'épaississant lentement, plus ou moins cornées. Des " tâches de charbon " brunes caractéristiques apparaissent sous les ongles, traduisant la présence de caillots thrombosiques dans les petits vaisseaux du lit de l'ongle. L'évolution à tendance verruqueuse accompagne en général un épaississement légèrement pachydermique du tégument des doigts. Cette forme, dans ce deuxième stade, peut persister longtemps telle quelle. Mais la nature clinique de la radiodermite peut être différente, c'est-à-dire scléro-atrophique, amincissant la peau qui se plaque aux plans profonds, rougit, est luisante, et contrairement à la forme précédente, est plus évolutive, et se complique plus vite d'ulcérations trophiques et atones, qui d'abord cicatrisent, puis récidivent et passent à la chronicité. Dans ces cas, les panaris péri-unguéaux sont fréquents, les ongles deviennent dystrophiques et déformés. Les verrues kératosiques, si elles sont volumineuses et situées sur les plis de flexion. se fissurent, saignent, sont douloureuses.

Enfin, plus tard survient la dégénérescence cancéreuse, sous deux masques possibles : l'un insidieux, tardif, lent, latent sous une verrue kératosique en général assez volumineuse et ancienne, et là, reste souvent une surprise ou une révélation de l'anatomie pathologique; ou bien l'autre masque, plus à découvert et moins inattendu, sous la forme plus précoce, plus vite révélée; d'un bourgeonnement saignant, parfois douloureux sur les bords d'une ulcération. Dans 
ma série, la dégénérescence maligne s'était faite dans plus de la moitié des cas $(55,76 \%$ ), elle semblait être survenue dans des délais très différents : en moyenne douze ans après l'apparition des premières lésions. Mais pour dire le caprice de cette éclosion, citons par exemple cette radiodermite aiguë que j'opérai un an après l'accident causal, et où l'examen histologique décela déjà deux cancers. Il s'agissait toujours d'épithélioma spino-cellulaire. La forme de cancer sous verrue, citée plus haut, kérato-cancer, apparaissait le plus souvent sur des peaux épaissies, était en général muette, d'évolution très lente, et assez bénigne, non infiltrante ou n'envahissant que tardivement les plans profonds, permettant ainsi une opération conservatrice du doigt, n'emportant que la peau. La deuxième forme : l'ulcéro-cancer, caractérisait plutôt les évolutions sur tégument scléroatrophique, était plus rapide, plus infiltrante, envahissant presque d'emblée les formations sous-jacentes, obligeant ainsi au sacrifice de la phalange ou du doigt.

Mais d'une façon générale, ce cancer radiodermique professionnel fut d'évolution locale et lente, et je constatai seulement trois fois un envahissement ganglionnaire, et encore celui-ci, curable à temps. Le cancer laisse, heureusement somme toute, de grandes possibilités chirurgicales et de sérieux espoirs de bon résultat.

Le taux de dégénérescence constaté dans ma statistique est plus élevé que celui des autres séries publiées. C'est probablement parce qu'il fut fait un débitage histologique systématique de toutes mes pièces d'exérèse; et aussi parce que les radiodermites digitales dégénèrent plus souvent que les autres, soumises qu'elles sont à des conditions différentes, propres à la malignisation : stimulation chronique professionnelle de petites irradiations répétées durant des années. Notons du reste que les examens furent faits par plusieurs anatomo-pathologistes, et que leurs constatations donnèrent les mêmes taux de dégénérescence.

En ce qui concerne les structures et les classements anatomo-pathologiques, et pour parler d'abord en quelques mots seulement des radiodermites aiguës, il est commode et rationnel d'assimiler celles-ci aux brûlures thermiques avec leurs trois degrés, érythémateux, épidermique, dermique.

Pour les radiodermites chroniques c'est le derme qui est le principal siège du drame, à cause de la primauté de la lésion des petits vaisseaux, qui va conditionner l'anoxie, puis la sclérose du collagène qui feutre le derme. Mais il existe aussi, et d'emblée, des dystrophies des cellules de la couche muqueuse de l'épiderme, premières graines du cancer à venir. Ces anomalies engendreront auparavant une évolution anormale des couches cornées de surface, origine des dyskératoses, d'où ces verrues très particulières aux radiodermites des doigts.

L'évolution et la classification histologique de ces lésions n'est pas fortement parallèle ni contemporaine des aspects cliniques, stade pour stade; des lésions atrophiques, pachydermiques, télangiectasiques, trophiques n'ont pas de personnalité microscopique. A l'inverse, des cancers intra-épidermiques ou souskératose peuvent être cliniquement muets. Les stades microscopiques sont :

I) d'abord de radiodermite simple, avec involution et atypies épidermiques;

2) puis de radiodermite dégénérée selon trois degrés :

a) intra-épidermique (précancéreux, ou degré initial ?);

b) envahissant, la membrane basale étant rompue, mais sans profondeur;

c) infiltrant où les plans sous-jacents sont pris.

vor. $6-\mathrm{N}^{\circ} 3$ 
On pourrait, dans le cas très spécial des radio-lésions ouvrir une discussion sur la signification de leur dégénérescence. Elle peut s'assimiler à celle des cicatrices et de tout vieillissement tissulaire artificiel, solaire par exemple. On peut aussi se demander si le cancer passe par un stade intra-épidermique obligatoire, ou s'il est directement envahissant. Ce qu'on sait des cancers in situ du col utérin donne à penser que ce stade intra-épidermique pourrait n'être qu'une étape vers l'invasion. Ici encore se pose le problème du seuil de la malignisation et du cancer pré-invasif intra-épidermique.

Quoi qu'il en soit, que le cancer soit sur une kératose ou sur les bords d'une ulcération, il est vraisemblable que le cancer est né d'une dysplasie des cellules du corps muqueux, ancienne et longtemps latente; mais dans le cas de l'ulcérocancer, il se peut aussi que la dégénérescence ait été la conséquence d'un ancien état inflammatoire chronique, comme il arrive sur de vieilles ulcérations ou cicatrices de brûlures.

Le problème du mécanisme intime de la transformation des radio-lésions est général et dépasse celui des doigts. Mais ce dernier a des caractères très personnels : on a invoqué le facteur irritatif des " taches de charbon " sous-unguéales ex de leur mécanisme d'expulsion. Je n'ai pas observé de fait clinique appuyant cette hypothèse; on invoque aussi avec plus de vraisemblance, les conséquences anoxiques et irritatives de la vascularité du derme, sur l'épiderme profond. Pour ma part, je ne puis m'empêcher de penser à une déviation, une viciation dysgénétique née de la première irradiation, à un " péché originel " portant en soi une vertu d'emblée dystrophiante de la cellule épithéliale du corps muqueux, " génie cancéreux " qui prépare dès ce premier jour sa dysplasie et son héritage dysgénétique à long terme, destiné soit à substituer tel quel, indéfiniment latent, somnolent, soit à se réveiller et à dégénérer sous l'influence de nouvelles irradiations. Les altérations du sous-sol dermique, la sclérose de celui-ci, son atrophie peuvent favoriser ce processus, mais je crois au primum movens de la dysplasie épithéliale d'emblée. Au demeurant le mécanisme peut être complexe ou non univoque, à la fois irritatif et dysplasique, c'est-à-dire dermique et épidermique, et selon les formes kératosique ou ulcéreuse.

Pour rester sur le plan de la pratique, et puisque la symptomatologie est impuissante à éliminer d'avance une dégénérescence, il faut que prudence et méfiance restent mère de sûreté. Aussi faut-il s'imposer très sévèrement la discipline du contrôle anatomo-pathologique extemporané en cours d'intervention de toutes les pièces excisées, qui guidera sur la tactique et l'étendue de l'opération, indiquant un état de bénignité ou de malignité, et dans ce dernier cas, la possibilité éventuelle de recoupes limitées, c'est-à-dire une excision conservatrice avec greffe, si la recoupe a largement passé en tissu sain, sinon, une désarticulation à minima (phalange ou doigt) si l'épithélioma est infiltrant. Cette pratique permet une chirurgie au maximum conservatrice.

Car le traitement des radiodermites des doigts, passé le premier stade d'atrophie-télangiectasie, est seulement chirurgical. Il n'y a pas de traitement médical. On ne peut en effet espérer de stabilisation définitive des premiers signes que durant cette période initiale, avec le renoncement définitif aussi à la pratique des rayons. Ainsi le médecin qui relève ces symptômes dispose d'un an environ pour se repentir de sa faute et ne plus y retomber. Ensuite, dès l'apparition des kératoses, des troubles atrophiques, l'évolution devient irréversible et appelle 
comme seul traitement l'excision chirurgicale du tégument lésé, peau et région unguéale atteintes, et son remplacement par une greffe de peau très mince prélevée de préférence sur l'avant-bras, ce qui est moins inconfortable. La prise de greffon est assurée en quelques heures, et il est important que la mobilisation active des doigts soit entreprise dès le $3^{\mathrm{e}}$ jour, avec leur usage pour de petits actes, manger, fumer, etc.

Moyennant l'active coopération toujours consentie par l'opéré et par son entourage, le séjour en clinique se réduit à onze jours en moyenne, la mobilité des doigts se récupère presque totalement en dix semaines, la sensibilité en six mois, la profession et la spécialité furent toujours reprises, par les médecins généralistes et les radiologues en vingt-cing jours en moyenne, par les chirurgiens en sept semaines, par les orthopédistes en neuf semaines. Leur constante récupération a une conséquence psychologique primordiale qui restitue aux opérés leur pleine confiance en l'avenir.

On informe toujours l'opéré de la possible survenue de nouvelles verrues en zones saines jusque là, qu'il faudra alors simplement coaguler. En ce qui concerne les cas dégénérés, j'ai dit plus haut la tactique adoptée : simple excisioncutanée et greffe pour les cancers non infiltrants, sacrifice minimum pour les autres, c'est-à-dire de la phalange ou au plus du doigt. Je n'ai observé que trois récidives et encore avant ma pratique de l'examen histologique extemporané per-opératoire. Un seul opéré fit, trois ans après, un cancer du poumon dont il finit par mourir, cancer peut-être métastatique de son cancer du doigt. La mortalité carcinologique serait donc de moins de un pour cent dans cette série.

Je ne répéterai jamais assez la morale de cette expérience :

- il ne faut jamais, et sous aucun prétexte, mettre les mains sous les rayons, même d'un amplificateur de brillance. « La meilleure protection contre un danger, c'est le refus du danger ") (SANTAGOSTINI I960); car il n'y a pas de protection préventive de la peau;

- il faut se repentir, c'est-à-dire s'abstenir, dès les premiers signes. Après eux, l'évolution est irréversible et l'aboutissement au cancer est inexorable si le temps lui en est laissé.

Le traitement chirurgical, lui seul, supprime les lésions; il conserve le doigt, grâce à l'excision-greffe, longtemps possible et suffisante, même avec un cancer, s'il est non infiltré; ce traitement simple donne des résultats fonctionnels et professionnels toujours bons. 
F. LAGROT

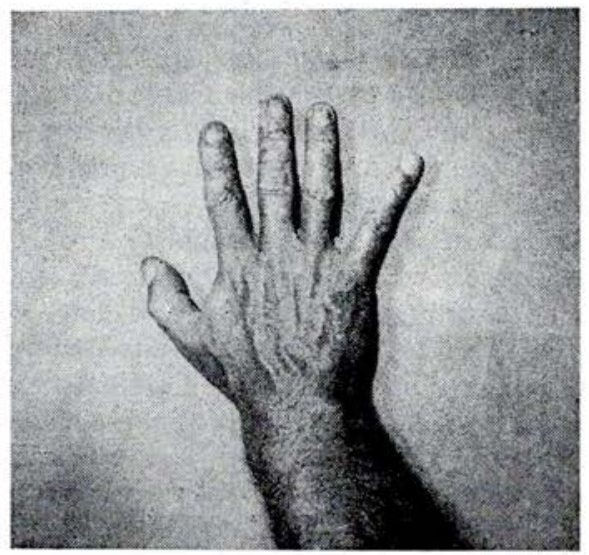

Fig. I A

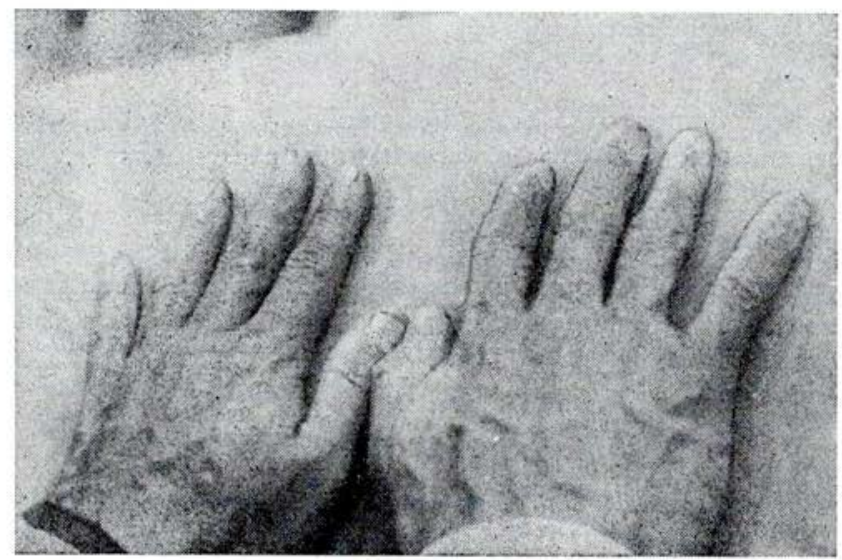

Fig. I B 

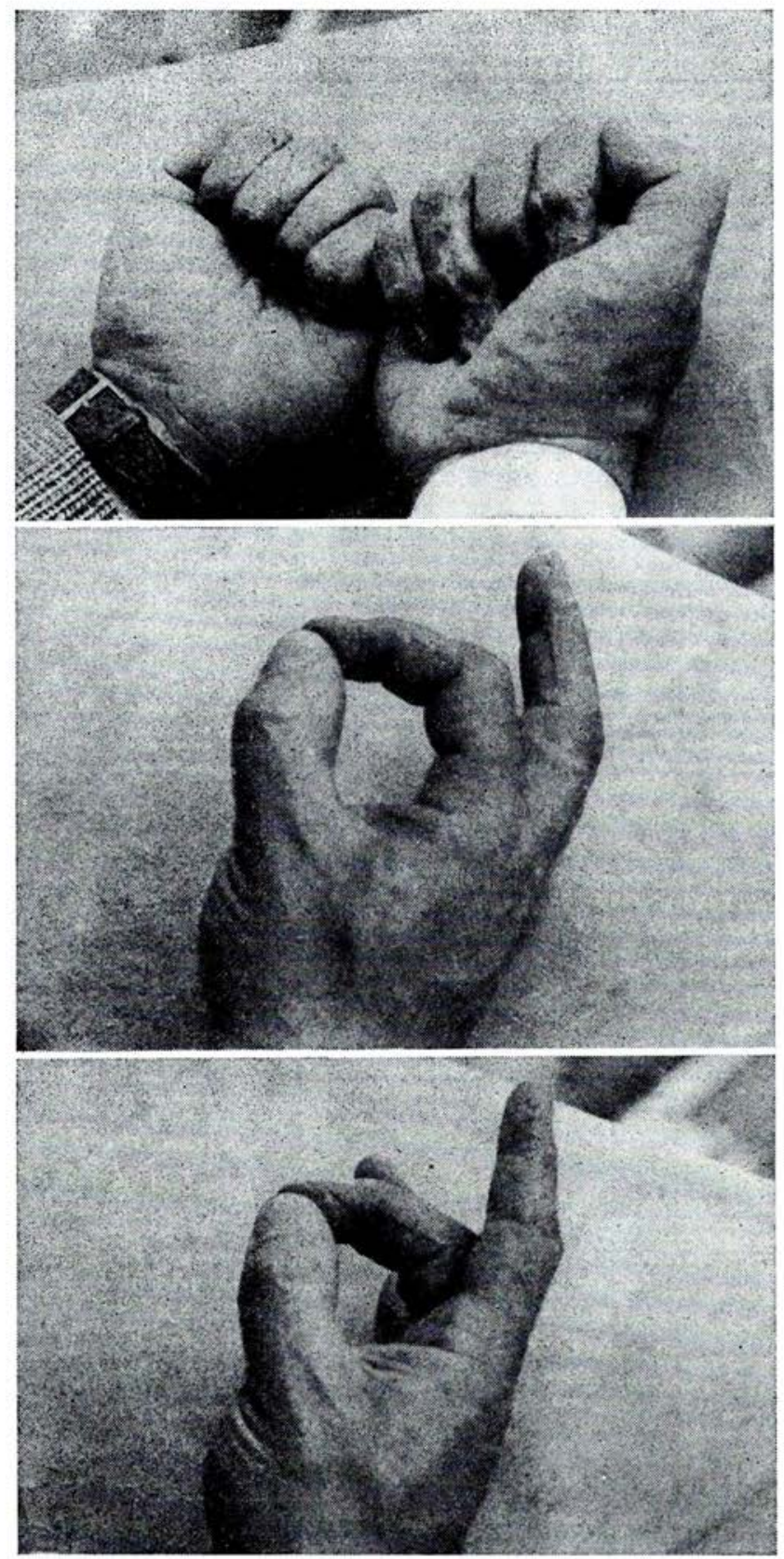

Fig. I. - A - Lésions $2^{\mathrm{e}}$ degré main droite. Excision-greffe B - C - D - E - Résultat au $15^{\mathrm{e}}$ mois.

Des lésions apparurent onze ans après sur la main gauche et furent opérées de la même façon. 


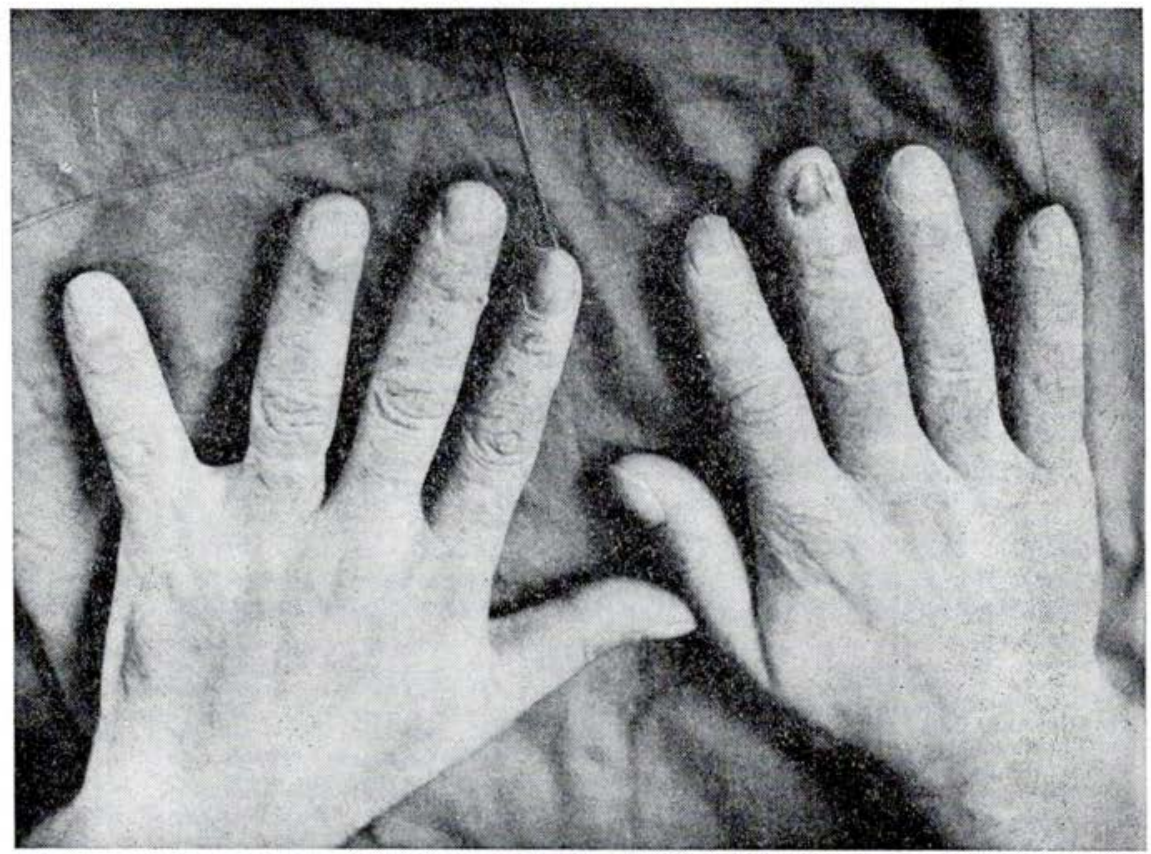

FIG. 2. - Lésions de stade II, de forme verruqueuse plus ou moins papillomateuse sur tégument à tendance pachydermique. Il fut pratiqué une excision-greffe.

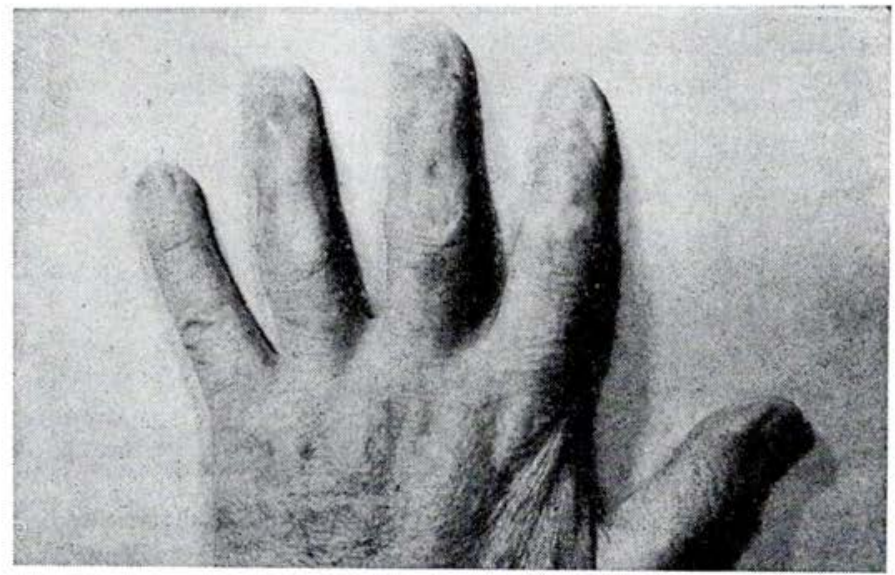

FIG. 3. - Atteinte des deux mains, lésions du $\mathrm{II}^{e}$ et du $I \mathrm{II}^{e}$ degré. Simple excision-greffe (A). 

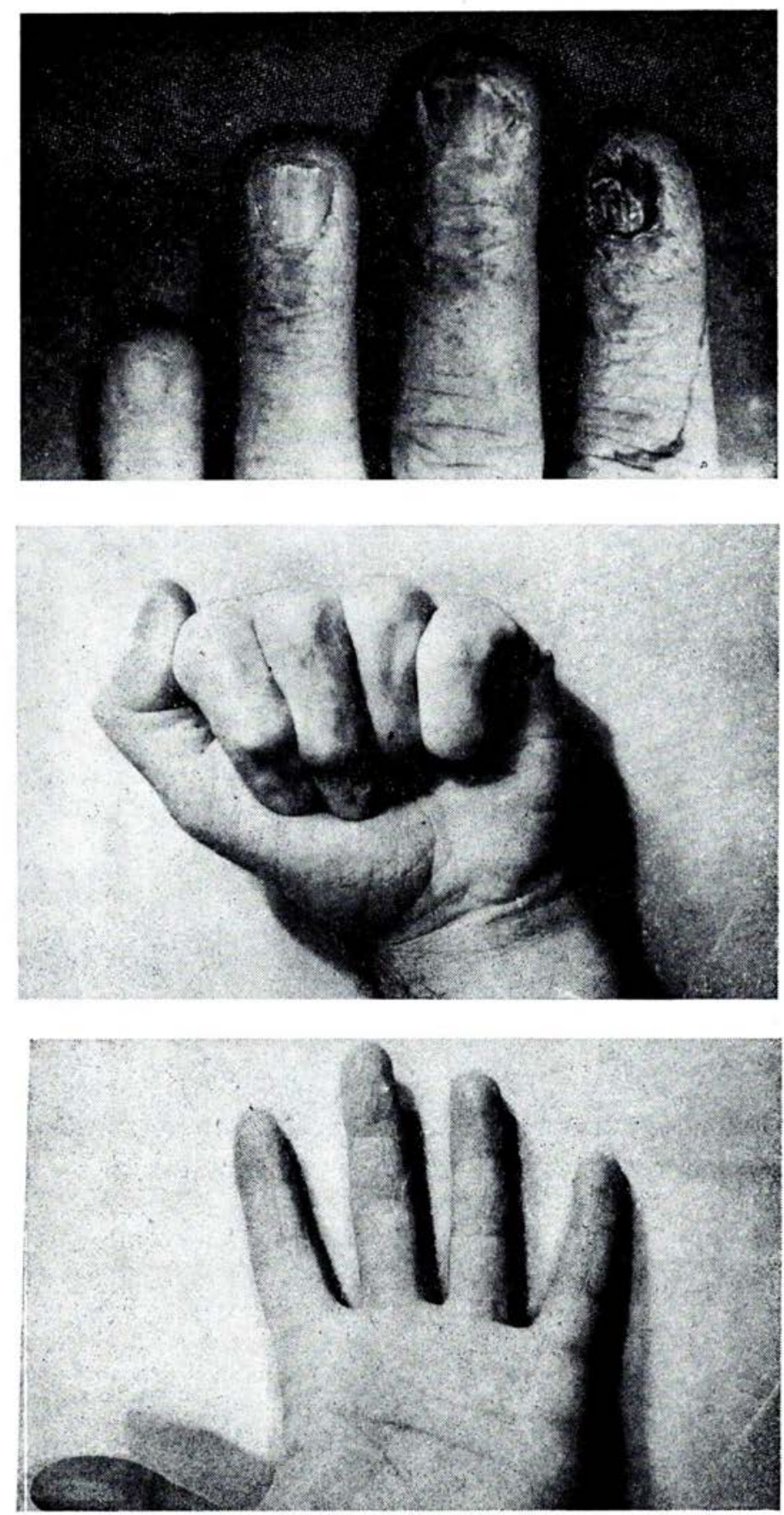

Fig. 3. - B - C - D. Résultat trois ans après; activité chirurgicale très importante. vol. $6-\mathrm{N}^{\circ} 3$ 


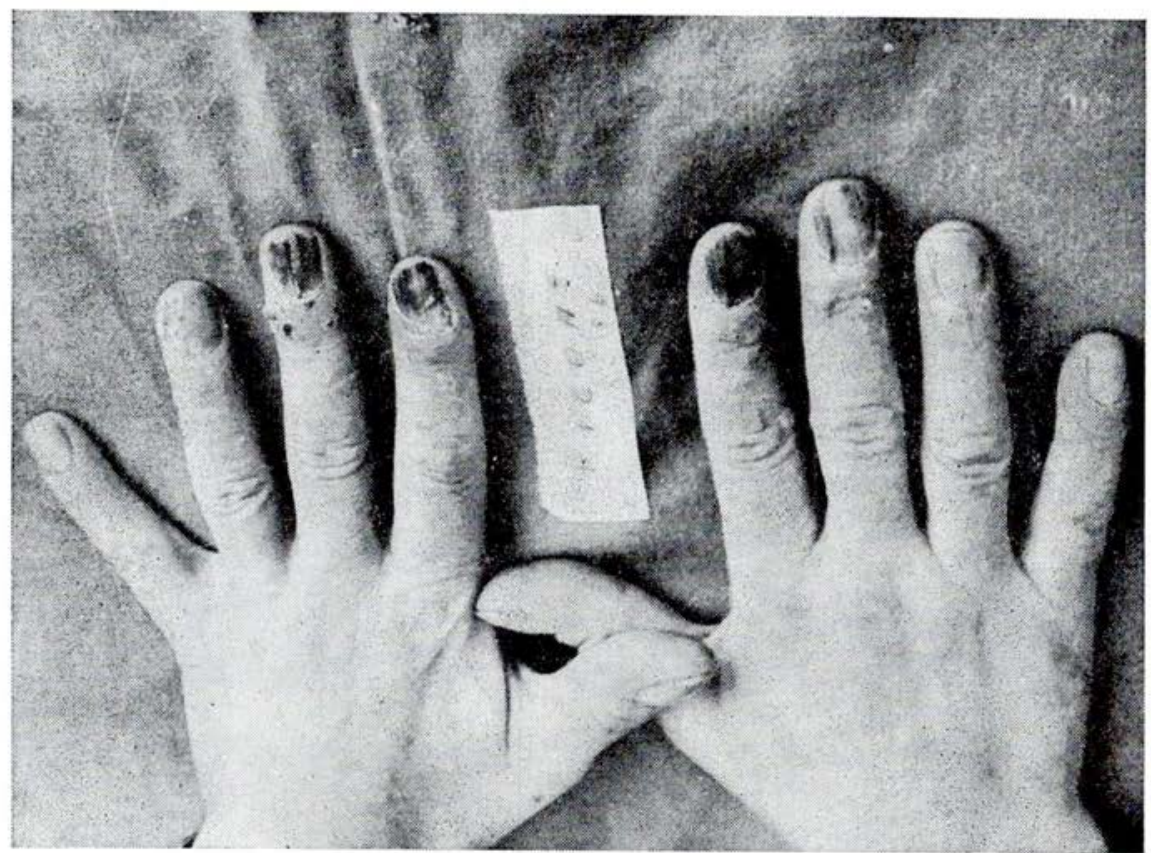

FIG. 4. - Stade IV. Cancers superficiels multiples, sous kératomes, du type Bowen. L'excision-greffe a suffi. 


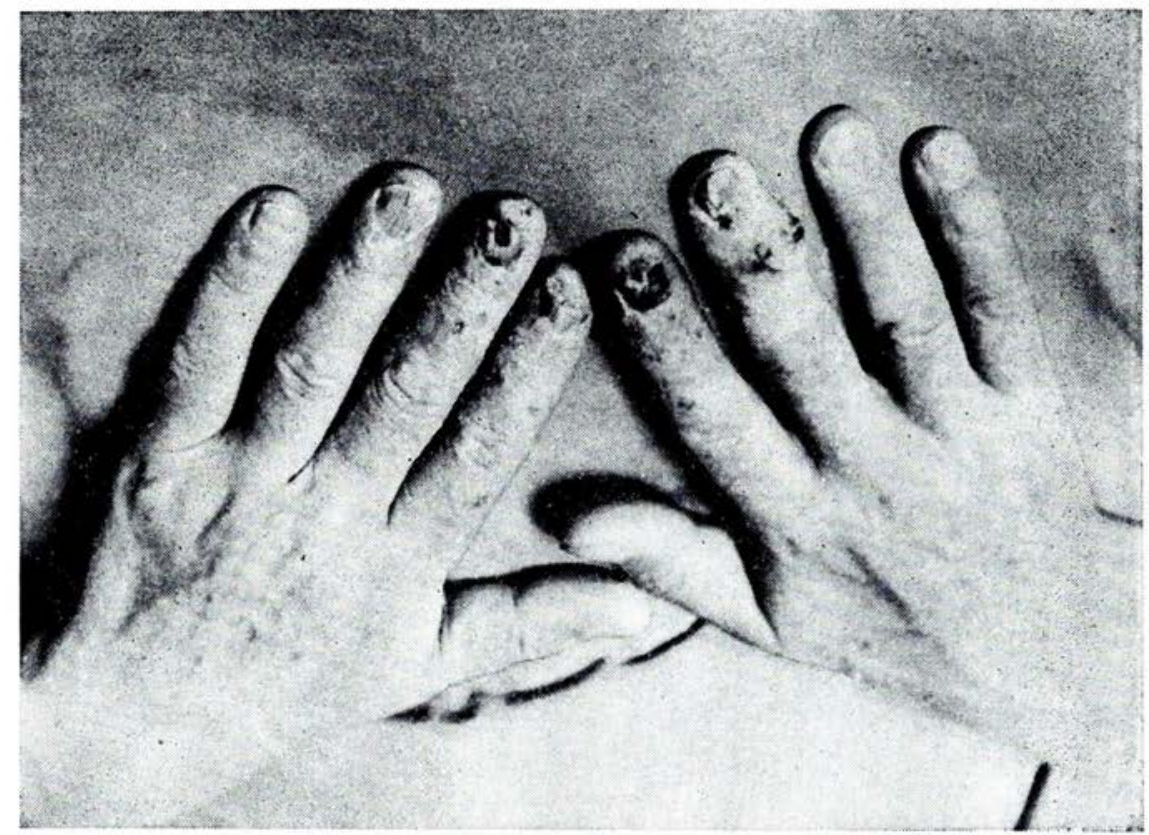

Frg. 5. - Sur la main gauche, stade III trophique. Simple excision-greffe.

Sur la main droite, épithélioma végétant sur ulcération de l'annulaire. Amputation des deux dernières phalanges

Guérison maintenue 12 ans après.

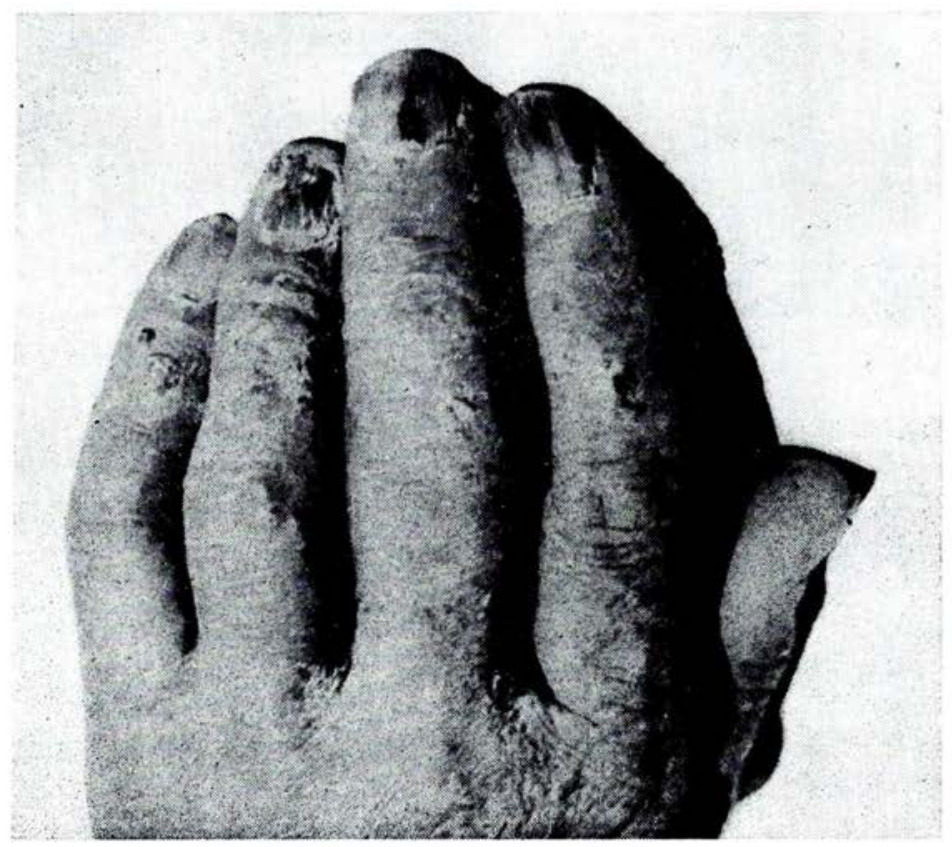

FIG. 6. - Stade IV, cancers multiples.

A (non invasifs à gauche, permettant une simple excision-greffe). 


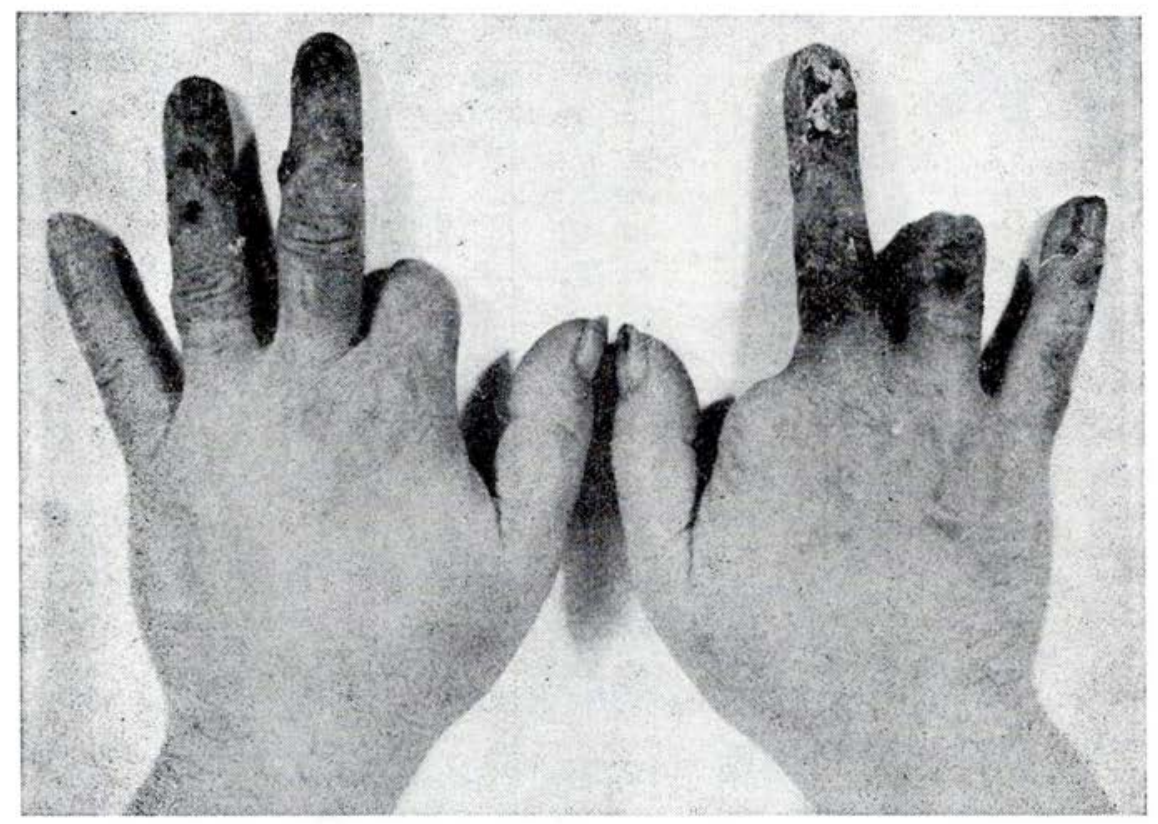

Fig. 7. - A. Stade IV, cancers infiltrants. Avait été traité par "plésiothérapie " et amputé partiellement de certains doigts. L'excision greffe fut suffisante pour les autres. La guérison de ces doigts opérés se maintient $\mathrm{I}_{4}$ ans après, mais une nouvelle lésion apparaît en zone saine à la base du médius gauche. Excision-greffe de cette lésion. 


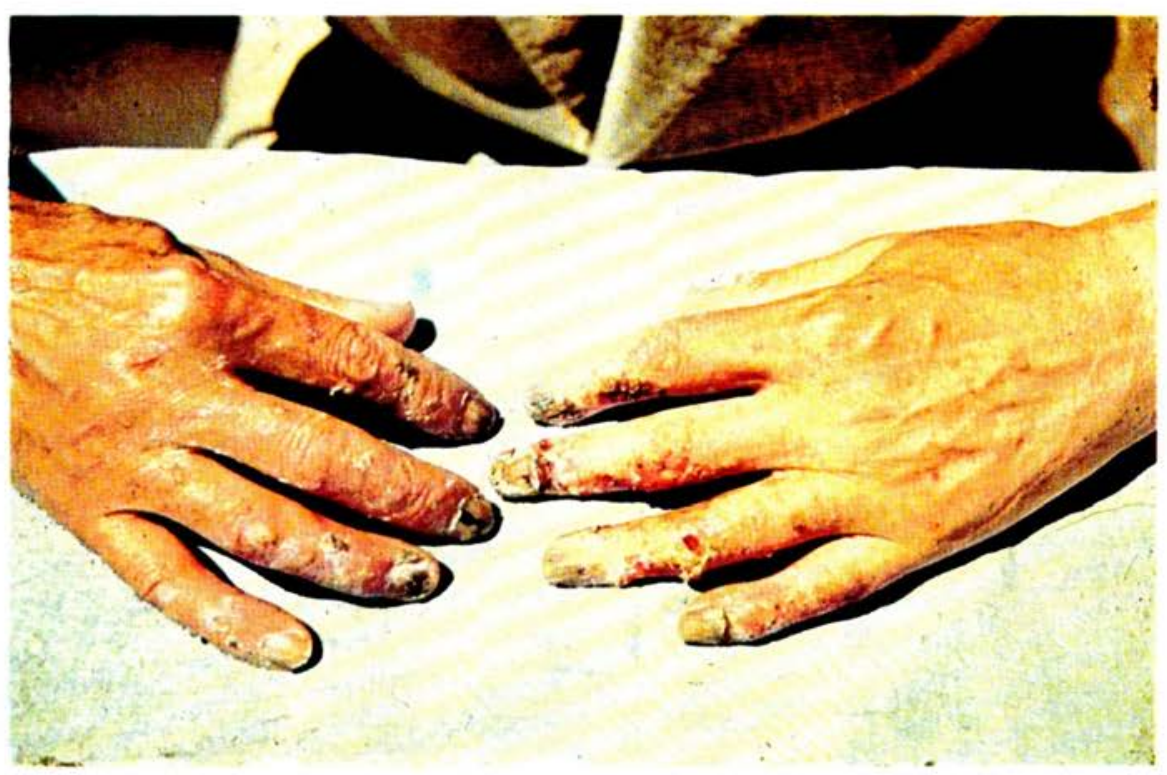

Fig. $6 \mathrm{~B}$ (coulcur)

Chirurgien, 65 ans. Cancers multiples infiltrants, sur forme scléroatrophique. Fut opéré il y a seize ans. Deux doigts furent amputés, les autres traités par excision et greffe. Le résultat se maintient à ce jour, sans récidive. 



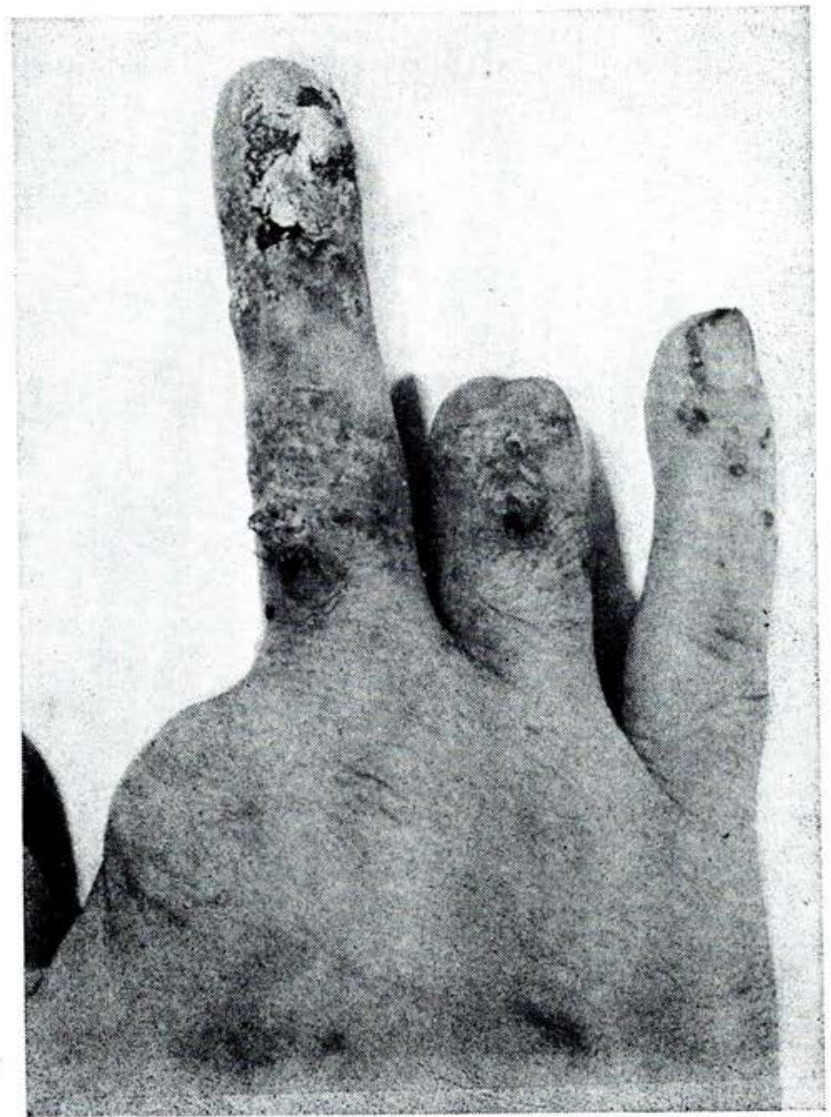

FIG. 7. - B. Id. détail.

voL. $6-\mathrm{N}^{\circ} 3$ 


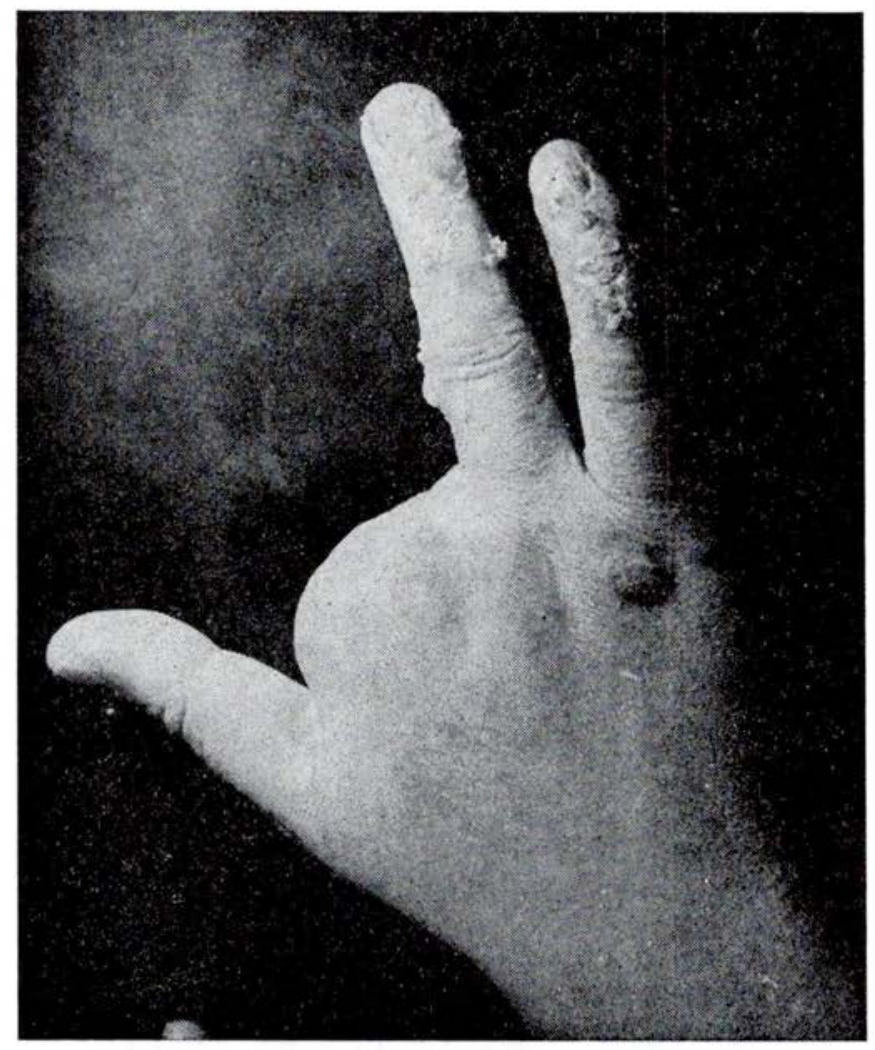

Fig. 8. - Stade IV, cancer invasif sous kératome. Médius : épithélioma, désarticulation de deux phalanges, annulaire : trois épithéliomas, désarticulation. 


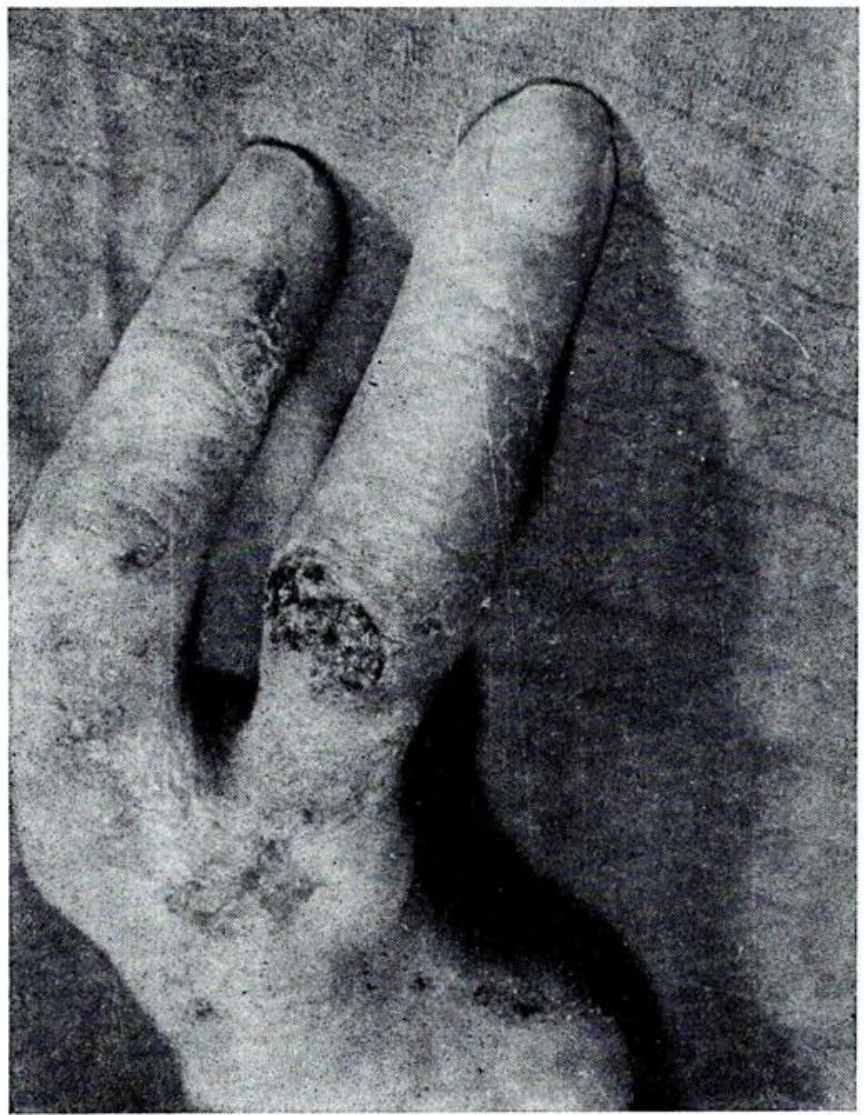

FIG. 9. - Cancer sur ulcération, chez une infirmière (une partie de la main avait déjà dû être amputée). Désarticulation du doigt atteint, et simple excision greffe des lésions du doigt restant.

L'autre main portait un vaste cancer dorsal qui exigea l'amputation du poignet.

Guérison maintenue 20 ans après.

vol. $6-\mathrm{N}^{\circ} 3$ 